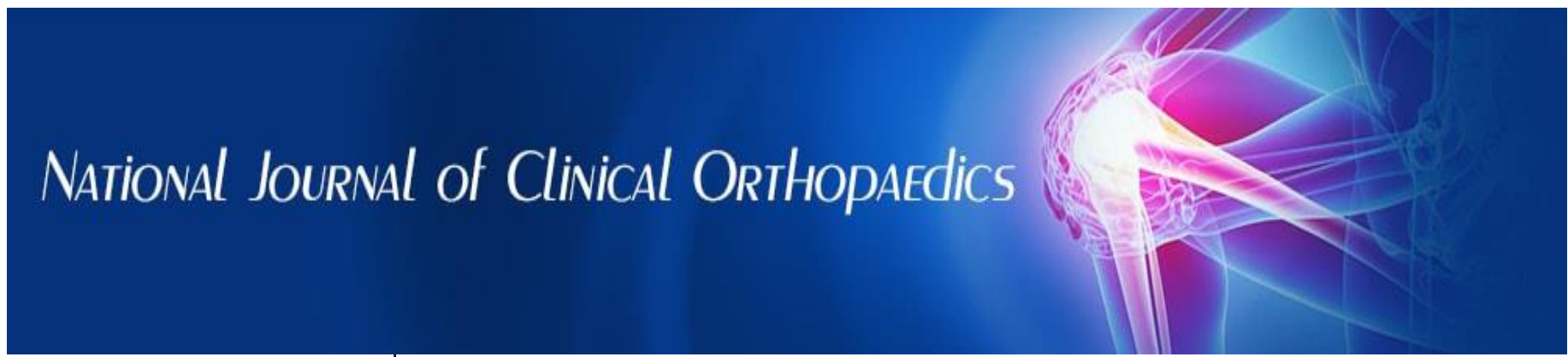

ISSN (P): 2521-3466

ISSN (E): 2521-3474

(C) Clinical Orthopaedics

www.orthoresearchjournal.com

2019; 3(3): 96-99

Received: 19-05-2019

Accepted: 21-06-2019

Mohammad Asimuddin

Associate Professor, Department

of Orthopaedics, Khaja

Bandanawaz Institute of Medical

Sciences (KBNIMS), Kalaburagi,

Karnataka, India

Marthand Kulkarni

Assistant Professor, KBNIMS,

Kalaburagi, Karnataka, India
Correspondence

Marthand Kulkarni

Assistant Professor, KBNIMS

Kalaburagi, Karnataka, India

\section{Open reduction and internal fixation with $k$-wire in type III supracondylar fracture of humerus in children by posterior approach}

\section{Mohammad Asimuddin and Marthand Kulkarni}

DOI: https://doi.org/10.33545/orthor.2019.v3.i3b.167

\begin{abstract}
Background: Supracondylar fractures of humerus are the most common fractures seen in children. Severely displaced supracondylar fractures of the distal humerus in children are a challenging problems. Displaced supracondylar fractures are notorious for difficulty in reduction, maintenance of reduction and involvement of neurovascular structures.

Open reduction and internal fixation is applied when closed reduction fails to give satisfactory reduction. The aim of the study was to determine the cosmetic and functional results following open reduction and internal fixation with k-wire for type III supracondylar fractures of the humerus by posterior approach.

Method: Twenty cases of type III supracondylar fractures of humerus were included in the study. The mean age of the patients was 8.4 yrs (ranges from 5-14 years). The male to female ratio was 2:1 and left side was involved in 14 cases and 6 had right sided injuries. All fractures were of extension type (gartland s type III). Open reduction and internal fixation with k-wire was performed by posterior approach. K-wires were removed between 3- 4 weeks post operatively and follow up was done at 6wks, 12 wks, 6 months and 12 months. The results were graded using the criteria described by Flynn et al.

Results: Excellent results were achieved in 11 pts (55\%), good in 4 pts (20\%), fair in 2 pts (10\%), while poor results were seen in 3 pts $(15 \%)$.

Conclusion: Open reduction and internal fixation with k-wires by posterior approach in type III supracondylar fractures of humerus in children is satisfactory.
\end{abstract}

Keywords: Type III supracondylar fractures, open reduction, internal fixation, posterior approach

\section{Introduction}

Supracondylar fractures of the humerus accounts for $60 \%$ of all fractures of the elbow in children. The rate of occurrence increases steadily in the first five years of life to peak at 5-7 years of age ${ }^{[1]}$. Supracondylar fractures of humerus is one of the commonest fractures in children. displaced supracondylar fracture of humerus have always presented a challenge in their management ${ }^{[2]}$. Displaced supracondylar fractures are notorious for difficulty in reduction, maintenance of reduction and frequent involvement of neurovascular structures ${ }^{[3]}$.

The management of displaced supracondylar fractures of the humerus is one of the most difficult of the many fractures seen in children ${ }^{[4]}$. Closed reduction with splints or cast immobilization has traditionally been recommended for displaced supracondylar fractures but loss of reduction and necessity of repeated manipulation is likely to go for mansion, produces varus or valgus deformity of elbow and elbow stiffness ${ }^{[5]}$. When a displaced supracondylar fracture cannot be reduced satisfactorily by closed means, open reduction and internal fixation is performed, maclellan strongly recommended open reduction for displaced fractures which cannot be reduced anatomically ${ }^{[6]}$. The aim of this study was to determine the functional outcome of type III displaced supracondylar fractures in children with open reduction and internal fixation with k-wire by posterior approach.

Materials and methods.

This study was conducted in orthopaedics department of khaja bandanawaz teaching and general hospital, kalaburagi, for a period of 1 year from Jan 2018 to dec 2018. The study received clearance from ethical committee of the institution. 
In this study twenty cases of displaced extension type of supracondylar fractures (gartland type III) of the humerus in children were treated by open reduction and internal fixation with k-wires by posterior approach.

\section{Inclusion criteria}

Age less than 14 years.

Open fractures

Fractures which cannot be reduced by closed reduction.

Fractures with neurovascular complications.

\section{Exclusion criteria}

Age more than 14 years.

Medically unfit patients.

A detailed history was elicited from the patients. The fracture pattern seen in the $\mathrm{x}$-ray was classified according to Gartland's classification ${ }^{[2]}$.

Type-I Non-displaced

Type-II Minimally displaced with intact posterior cortex

Type-III Completely displaced with no cortical contact

a) Posteromedial

b) Posterolateral

Before surgery necessary laboratory investigation were done.

\section{Operative technique.}

With the patient under general anesthesia and in prone position with the elbow supported on the sand bag, extremity was prepared from axilla to wrist \& draped. With a posterior Campbell's approach, (fig-1) ulnar nerve was isolated, an inverted tongue shaped incision was made over the triceps. All the blood clots \& debris removed from the fracture site. Fracture was reduced $\&$ internally fixed with two or three smooth crossed Kirschner's wire of diameter 1.5 to $2.5 \mathrm{~mm}$. The pins were introduced with the help of a hand drill. The lateral wire was introduced through the anterior side of lateral condyle and directed posteriorly into the posteromedial side of the opposite cortex. The medial wire was started through the posteromedial side of medial condyle and engaged into the anterolateral side of opposite cortex. While introducing the medial wire, greater care was taken to avoid the ulnar nerve. By this method, the wires were laid high above the fracture site. The pins were cut percutaneous for easy removal later. After the pins are placed, the elbow is extended and the carrying angle is measured and compared to that on the non-affected side. The stability of the fracture was checked. Then the wound was closed and a drain wan kept and a posterior splint was applied with the elbow in $90^{\circ}$ flexion and forearm in neutral rotation.

\section{Postoperative Course}

- Full arm posterior slab was used, cuff and collar was given. The limb was elevated.

- Antibiotics Analgesics and anti-inflammatory drugs were given in post-operative period.
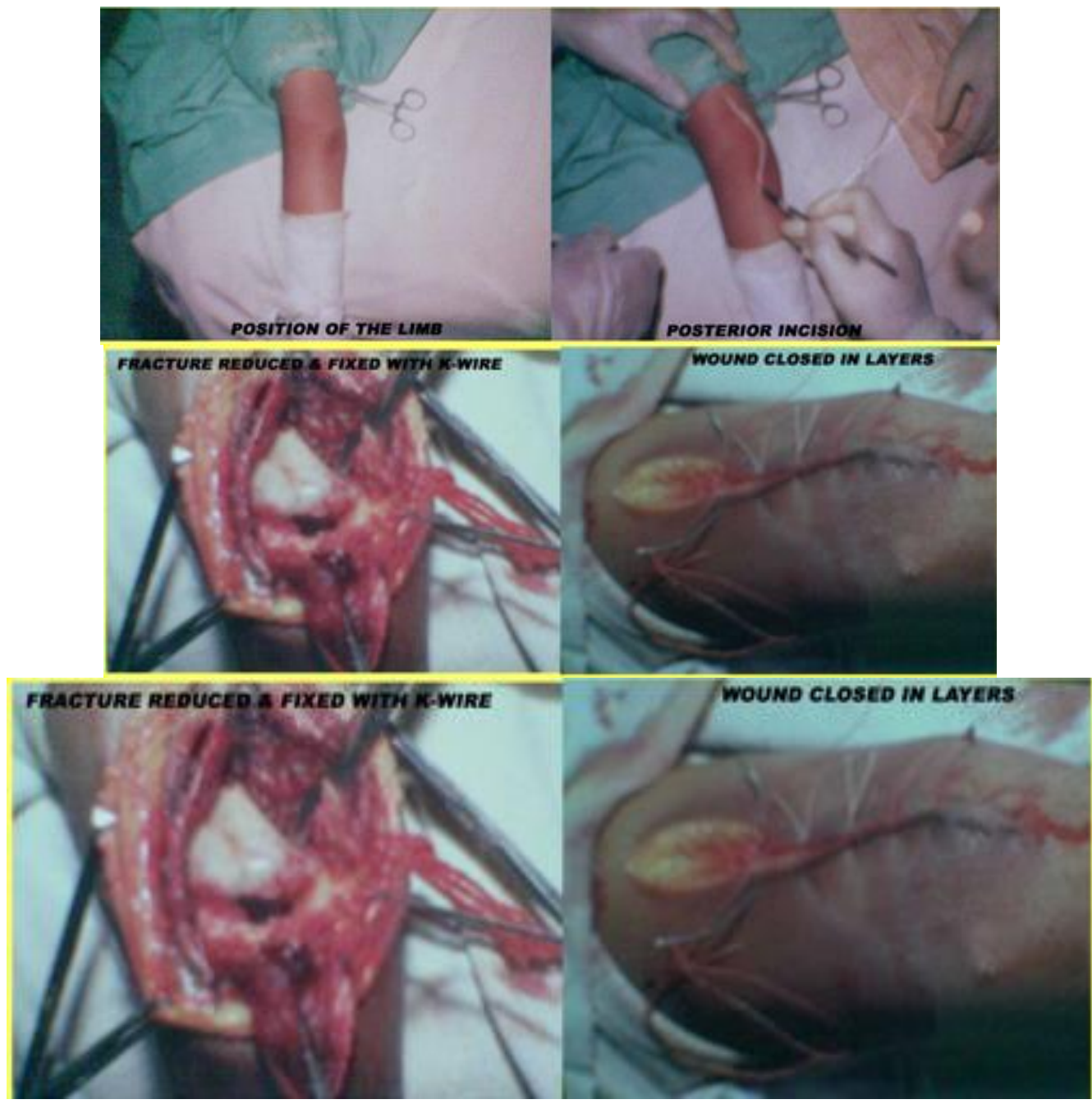

Fig 1: ORIF by posterior approach 
Advice given at the time of discharge

a) Elevation of the limb till the edema subsides.

b) Active mobilization of fingers and shoulder joint.

c) Patients were called at third week for " $K$ " wire removal. After the k-wires were removed the posterior slab was discarded, and active movements of elbow were started. Special mention was made to avoid oil massage and passive stretching which is advocated by unqualified medical personnel. All these cases were advised to attend the outpatient department at regular intervals of 3 weeks, 6 weeks, 3 months, 6 months and 12 months for checkup and to note down the progress of union, range of movement at elbow and onset of any deformity. Range of movements and carrying angle were measured using goniometer.

Check X-ray were taken postoperatively at the end of 3-4 weeks, 3 months and 6 months. Cases were followed from 6 to 12 months.

The final results were evaluated by Flynn's criteria. ${ }^{7}$ Table -1

Table 1: Flynn's Grading System

\begin{tabular}{|c|c|c|c|}
\hline Results & Rating & Cosmetic factor: Carrying angle loss (degrees) & Functional factor: Loss of range of motion (degrees) \\
\hline & Excellent & $0-5$ & $0-5$ \\
\hline Satisfactory & Good & $6-10$ & $6-10$ \\
\hline & Fair & $11-15$ & $11-15$ \\
\hline Unsatisfactory & Poor & Over 15 & Over 15 \\
\hline
\end{tabular}

The results were graded as excellent, good, fair and poor according to loss of range of motion and loss of carrying angle. Cases were followed up to 12 months.

\section{Results}

In the present study, the age of the patient ranged from 5-14 years, with a mean age of 8.4 yrs. In our study, the major cause of fracture was fall on outstretched hand. Of the 20 patients, 13 had posteromedial and 7 had poster lateral displacement.
In our study, average interval between injury and surgery was 2.3 days (2-3 days). There were 3 cases of superficial pin tract infection, which were treated by antibiotics. we had 2 cases of iatrogenic ulnar nerve palsy which recovered completely within 5-6 months. In our study there were 2 cases of cubits varus.

\section{Carrying angle loss}

At the final follow up more than $15^{\circ}$ carrying angle loss was noted in 2 patients $(10 \%)$. Table -2

Table 2: Status of loss of carrying angle

\begin{tabular}{|c|c|}
\hline Carrying angle loss & No. of cases (\%) \\
\hline $0-5$ & $12(60 \%)$ \\
\hline $6-10$ & $4(20 \%)$ \\
\hline $11-15$ & $2(10 \%)$ \\
\hline$>15$ & $2(10 \%)$ \\
\hline
\end{tabular}

\section{Loss of range of motion}

noted in 3 patients (15\%). Table -3

At the final follow up more than $15^{0}$ loss of range of motion was

Table 3: Status of loss of motion

\begin{tabular}{|c|c|}
\hline Loss of range of motion (Degree) & No. of cases (\%) \\
\hline $0-5$ & $11(55 \%)$ \\
\hline $6-10$ & $4(20 \%)$ \\
\hline $11-15$ & $2(10 \%)$ \\
\hline$>15$ & $3(15 \%)$ \\
\hline
\end{tabular}

\section{Functional results}

Final results were evaluated by Flynn's criteria. Table -4

In our study 17 patients $(85 \%)$ had satisfactory results. and 3 patients $(15 \%)$ had unsatisfactory results which were rated as poor. The poor results were due to cubitus varus deformity and due to elbow stiffness in two patients and in one patient due to loss of reduction.

Table 4: Functional results based on flynn"s criteria

\begin{tabular}{|c|c|c|c|}
\hline Treatment Outcome & Rating & Loss of movement and carrying angle (Degree) & No. of cases $(\%)$ \\
\hline \multirow{3}{*}{ Satisfactory } & Excellent & $0-5$ & $11(15 \%)$ \\
\cline { 2 - 4 } & Good & $6-10$ & $4(20 \%)$ \\
\cline { 2 - 4 } & Poor & $11-15$ & $2(10 \%)$ \\
\hline Unsatisfactory & Poor & $>15$ & $3(15 \%)$ \\
\hline
\end{tabular}

\section{Discussion}

Supracondylar fracture of humerus is a common injury in children around elbow ${ }^{[8]}$.

Many methods have been proposed for the treatment of displaced supracondylar fracture of humerus in children, such as closed reduction and plaster of paris slab application, closed reduction and percutaneous pinning, open reduction and internal fixation.

Kasser et al. considered closed reduction and percutaneous pinning as the treatment of choice ${ }^{[8]}$.

In developing countries due to non-availability of image intensifier and late presentation preludes the advantage of closed 
reduction and percutaneous pinning.

The main goal of operative treatment of supracondylar fracture is to safely create an adequately stable construct to prevent axial rotation and coronal or sagittal tilt to avoid post-operative deformity, internal rotation of the distal fragment is the major predisposing factor to varus deformity ${ }^{[9]}$.

Repeated attempts of closed reduction fails because of soft tissue interposition in approximately $25 \%$ of the cases.closed reduction fails because of muscle interposition with a varus malposition [10].

Some authors have advocated medial or lateral approach depending on the type of displacement ${ }^{[9]}$.

Open reduction is indicated if closed reduction fails and in cases with neurovascular damage ${ }^{[11]}$. The final results were evaluated by Flynn, s criteria. In our study 3 patients (15\%) had unsatisfactory results. Pirone et al reported 22\% unsatisfactory results by open reduction and internal fixation method. ${ }^{[12]}$

Philip et al reported $82 \%$ excellent or good results, $12 \%$ fair and $6 \%$ poor results in open reduction of irreducible supracondylar fractures ${ }^{[13]}$.

Zionts et al. reported $78 \%$ excellent results in open reduction and internal fixation. We reported $55 \%$ excellent results in our series ${ }^{[14]}$.

In our current study we had used posterior approach which is safe, simple and accuracy of reduction is ensured. By open reduction haematoma is released and so myositis ossificans is eliminated. Wilkins reported a $1.4 \%$ incidence of myositis ossificans and $0 \%$ incidence of lasting neurovascular deficits ${ }^{[9]}$. In our study we had no complication of myositis ossificans. When the fracture is complicated by neurovascular injury this approach is not suitable.

\section{Conclusion}

From our study we conclude that open reduction and internal fixation with k-wire for type III supracondylar fracture of humerus in children is efficient and succesfull treatment method when closed reduction fails.

\section{References}

1. D Ambrosia RD. Supracondylar fractures of humerusprevention of cubitus varus. J Bone and Joint Surg (Am). 1972; 54:61-6.

2. Gartland JJ. Management of supracondylar fractures of the humerus in children. Surg Gynaecol Obstet. 1959; 109:14554.

3. Bachman D, Santora S. Orthopaedic trauma. In: Fleisher GR, Ludwigs S, editors. Textbook of Paediatrics Emergency Medicine. Philadelphia.

4. Wolters Kluwer/Lippincott Williams \& Wilkins Health, 2006.

5. Haddad RJ, Saer JK, Riordan DC. Percutaneous pinning of displaced supracondylar fractures of the elbow in children. Clin Orthop. 1970; 71:112-7.

6. Canale TS. Fractures and dislocations in children. In: Campbell's Operative Orthopaedics. Volume 3. 9th Edition. New York: Mosby. 1998, 2407-2422.

7. MacLennan A. Common fractures around the elbow in children. Surg Gynecol Obstet. 1937; 64:447-453.

8. Flynn JC, Mathews JG, Benoit RL. Blind pinning of displaced supracondylar fractures of the humerus in children. J Bone \& Joint Surg (Am). 1974; 56:263-272.

9. Kasser JR, Beaty JH. (Eds.). Supracondylar fractures of the distal humerus (Chapter14). In: Rockwood and Wilkins Fractures in Children. 6th edition, Lippincott Williams and
Wilkins: Philadelphia, 2006, 543-89.

10. Wilkins KE. Fractures and dislocations of the elbow region. In: Rockwood CA, Wilkins KE, King RE, eds. Fractures in Children. 3rd ed. New York: JB: Lippincott, 1991, 509-28.

11. Farnsworth C, silva P, Mubarak S. Etiology of supracondylar humerus fracture. J pediatr orthop 1988; 18:38.

12. Reich RS. Treatment of intercondylar fractures of the elbow by means of traction. J Bone Joint Surg Am. 1936; 18:9971004.

13. Pirone AM, Graham HK, Krajbich JI. Management of displaced extension-type supracondylar fractures of the

14. humerus in children. J Bone Joint Surg Am. 1988; 70(5):641-50.

15. Philip FC, William Mc, Mervyn L. An Analysis of open reduction of irreducible supracondylar fractures of the humerus in children. Canad J Surg. 1998; 41(2):112-18.

16. Zionts LE, Mekellop HA, Hathaway R. torsional strength of pin configuration used to fix supracondylar fractures of the humerus in children. J Bone. Joint. Surg. AM. 1994; 76(2):253-6. 